\title{
Ape metaphysics: Object individuation without language
}

\author{
Natacha Mendes *, Hannes Rakoczy, Josep Call \\ Max-Planck-Institute for Evolutionary Anthropology, Department of Developmental and \\ Comparative Psychology, Deutscher Platz 6, D-04103 Leipzig, Germany
}

Received 29 November 2005; revised 12 April 2007; accepted 13 April 2007

\begin{abstract}
Developmental research suggests that whereas very young infants individuate objects purely on spatiotemporal grounds, from (at latest) around 1 year of age children are capable of individuating objects according to the kind they belong to and the properties they instantiate. As the latter ability has been found to correlate with language, some have speculated whether it might be essentially language dependent and therefore uniquely human. Existing studies with non-human primates seem to speak against this hypothesis, but fail to present conclusive evidence due to methodological shortcomings. In the present experiments we set out to test non-linguistic object individuation in three great ape species with a refined manual search methodology. Experiment 1 tested for spatiotemporal object individuation: Subjects saw 1 or 2 objects simultaneously being placed inside a box in which they could reach, and then in both conditions only found 1 object. After retrieval of the 1 object, subjects reached again significantly more often when they had seen 2 than when they had seen 1 object. Experiment 2 tested for object individuation according to property/kind information only: Subjects saw 1 object being placed inside the box, and then either found that object (expected) or an object of a different kind (unexpected). Analogously to Experiment 1, after retrieval of the 1 object, subjects reached again significantly more often in the unexpected than in the expected condition. These results thus confirm previous findings suggesting that individuating objects according to their property/kind is neither uniquely human nor essentially language dependent. It remains to be seen, however, whether this kind of object individuation requires sortal
\end{abstract}

\footnotetext{
* Corresponding author. Tel.: +49 (0) 3413550 424; fax: +49 (0) 3413550444.

E-mail address: mendes@eva.mpg.de (N. Mendes).
} 
concepts as human linguistic thinkers use them, or whether some simpler form of tracking properties is sufficient.

(c) 2007 Elsevier B.V. All rights reserved.

Keywords: Object individuation; Non-human primates

\section{Introduction}

The world as we adult humans think of it consists to a large degree of individuals. Necessarily, we perceive and think of the objects that make up our world as objects of certain kinds - this tree, that chair, the person over there. Concepts such as "chair" or "tree" which indicate what kind of thing an individual is are called "sortal concepts" (Strawson, 1959). Sortal concepts, and only sortal concepts, supply the necessary criteria for quantification ("How many trees/chairs/ persons are there in this room?" - "How many objects?") and numerical identity ("Is this the same tree/ chair/person as the one I saw yesterday?" - again, "Is it the same thing?"). As there is no "entity without identity" (Quine, 1981, p. 102), picking out objects under sortals is a prerequisite for full-fledged objective reference to and thought about objects in the first place (Evans, 1982; Strawson, 1959).

Without sortal concepts, two kinds of more primitive thought about objects are possible: spatiotemporal tracking and feature placing. Spatiotemporal tracking (of what we as adults take to be specific objects) in roughly the following rather unspecific way: "This bounded piece of stuff that moves coherently and continuously through space and time" - what has been called in developmental psychology "Spelke object", and "proto-object" in research on visual attention (see, e.g., Pylyshyn, 2000). This kind of indexical tracking lacks the logical structure of object related thought proper as it cannot supply determinate criteria for numerical identity and quantification (neither the question "How many Spelke objects are in this room?" nor the question "Is this Spelke object (a statue made of clay) the same as the Spelke object that was here a second ago (a raw piece of clay before a statue was made out of it)?" have clear meaning; see Wiggins, 1997). ${ }^{1}$

A second kind of simpler thought about objects is feature placing (Strawson, 1959), which consists of registering of features and clusters of features, such as color, size etc. Feature placing has the following logical form: though features of objects are registered, they are not predicated of an object. "(There is) red-ness and roundness here"; "(It is) cold here", are paradigm cases of feature placing. Feature placing

\footnotetext{
${ }^{1}$ This is not to deny, of course, that we often do engage in such merely spatiotemporal tracking (particularly upon encountering objects of unknown kinds), and that such tracking can supply some pragmatically useful though non-determinate criteria for individuation (see Xu, 1997). This does not mean, however, that "Spelke object" is a simple sortal, or even the ontogenetically prior one, for the simple reason that it is not a sortal at all (see Wiggins, 1997).
} 
thought thus lacks the propositional structure of full-fledged thought as the registered features are not predicated of proper objects picked out with sortal concepts. ${ }^{2}$

The phylogeny and ontogeny of these different kinds of object-related thought have been of great interest in cognitive science in recent years. In cognitive developmental psychology, Xu and Carey (1996) discovered a striking ontogenetic shift at around 1 year. While human infants early in their first year are certainly capable of feature-placing, and have been shown to parse the world into "Spelke objects" in many habituation studies (e.g., Baillargeon, 1987; Spelke, Kestenbaum, Simons, \& Wein, 1995; for an overview, see Spelke, 1990), only around 12 months seemed infants in Xu and Carey's studies capable of individuating objects qua objects of a certain kind. In these studies, infants were habituated to a display where two different toys disappeared behind an occluder. In the "spatiotemporal" conditions, both toys were simultaneously visible when going behind the screen. In the "property/ kind" condition, in contrast, one toy (e.g., a duck) went behind the screen first, then a second, different toy (e.g., a truck) emerged from behind the screen and then disappeared there again. In the test phase the occluder was lifted and either only one of the toys was there (unexpected) or the two original toys (expected). That is, equipped with the ability to track "Spelke objects" one can succeed in the spatiotemporal condition ("success" meaning here: looking longer at the unexpected compared to the expected event). However, to succeed in the "property/kind" condition, tracking "Spelke objects" does not help, as there are no simultaneous spatiotemporal cues as to how many objects there are. Rather, this condition requires, $\mathrm{Xu}$ and Carey argued, understanding the numerical identity of the objects which are fixed under sortals ("this truck" and "that duck"). Xu and Carey found that 10-month-olds only succeeded in the spatiotemporal condition, but not in the property/kind condition whereas the 12-month-olds succeeded in both conditions. In subsequent studies with different stimuli (infants had to track people versus inanimate objects), the shift to kind-based individuation was found earlier at 10 months (Bonatti, Frot, Zangl, \& Mehler, 2002). Studies with an analogous but simplified methodology as $\mathrm{Xu}$ and Carey (1996) also found infant success in property/kind conditions somewhat earlier at 9.5 months (Wilcox \& Baillargeon, 1998; Wilcox \& Chapa, 2002), in some cases even at 5.5 months (Wilcox \& Schweinle, 2002). ${ }^{3}$

Converging evidence for a shift in object-related cognition comes from infant studies with methods other than habituation: In a manual search paradigm, Van de Walle, Carey, and Prevor (2000) found a similar developmental pattern. Infants in these studies were presented with a box into which two toys went in ways analo-

\footnotetext{
${ }^{2}$ For a good overview of these different kinds of object-related perception and thought, see Clark (2004).

${ }^{3}$ Though there has been an ongoing controversy about the interpretation of the studies showing earlier competence (in particular, see the debate between Needham and Baillargeon (2000) and Xu and Carey (2000) in Cognition). While Needham and Baillargeon (2000) and Wilcox and Schweinle (2002) argue that these studies reveal infants' true competence whereas the Xu and Carey (1996) findings underestimate early competence, $\mathrm{Xu}$ and Carey (2000) doubt whether the simplified studies really require kind-based object individuation. We return to the question what kind of evidence we would need for kind-based individuation in infancy in Section 4.
} 
gous to the procedure of the habitation studies. Again, there were a "spatiotemporal" and a "property/kind" condition. In both conditions, infants were allowed to search the box in the test phase and either found both objects (expected) or only one of them (unexpected). The dependent measure was whether and to which degree infants continued searching. Again, 12-month-olds searched longer after unexpected than after expected events in both conditions, whereas 10-month-olds did so only in the "spatiotemporal" condition. As in the case of the habituation studies, with an analogous but simplified manual search methodology (infant sees object of kind A go in the box, and then either finds this object or another object of kind B) the shift was found somewhat earlier at 9.5 months (Xu \& Baker, 2005).

Some further interesting findings in this area concern the relation of object individuation and language. First, Xu and Carey (1996) found that success in the "property/kind" condition was positively correlated with noun comprehension. Second, $\mathrm{Xu}$ (2002) found that younger infants (at 9 months) could succeed in the "property/kind" condition if both objects were verbally referred to with a sortal label ("Look a duck!" at time 1, and "Look, a truck!" at time 2). Third, Xu, Cote, and Baker (2005) used a variant of the manual search paradigm of Van de Walle et al. (2000). However, rather than seeing objects go in and out of the box, they just saw an experimenter who looked into the box twice, and either said twice "Wow! An X (novel noun)!" or said "Wow! An X" on the first look and then "Wow! A Y!" on the second look. Infants then searched the box and only found one object. The 12-month-olds in this study subsequently searched longer and more persistently when they had heard two than when they had heard only one label.

Based on these findings, $\mathrm{Xu}$ (2002) has suggested that object individuation according to kinds might be uniquely human because it is linguistically mediated. In particular, the suggestion goes, the acquisition of count nouns, which serve as "essence placeholders", enables an essentialist ontology of individuals. This suggestion fits with a long tradition in analytical philosophy which views the acquisition of sortal terms and quantifiers as the entrance into thought proper about objects, and which holds that without language the only possibilities of perceiving objects are featureplacing and spatiotemporal tracking (e.g., Davidson, 1982; Dummett, 1993; Quine, 1960; Sellars, 1963). Now, on the premise that the tasks used with infants (both the original habituation task by Xu and Carey (1996), the original search task by Van de Walle et al. (2000), and the simplified search task by Xu and Baker (2005)) measure kind-based object individuation (which we accept for now, following previous work in the literature, but see Section 4), the question whether this type of object individuation is uniquely human is an empirically testable one.

Three studies so far have tested non-human animals' property/kind-based object individuation. In a preliminary study, Uller, Carey, Hauser, and Xu (1997) used Xu and Carey's (1996) habituation methodology with rhesus monkeys and found that the monkeys succeeded on both spatiotemporal and property/kind trials. Santos, Sulkowski, Spaepen, and Hauser (2002), in a manual search study, tested rhesus monkeys with a methodology similar to the one used by Van de Walle et al. (2000) and Xu and Baker (2005). In the spatiotemporal conditions, monkeys always found one-object in a box. Before, they had either seen one object go into the box 
(expected condition), or two spatiotemporally distinguishable objects go into the box sequentially (unexpected condition). In the property/kind conditions, monkeys saw an object of kind A go into the box, and then either found that object (expected) or an object of a different kind/with different properties (unexpected). Analogous to the infant studies, monkeys were found to search longer after unexpected than after expected events in both conditions. Phillips and Santos (2007) also tested rhesus macaques, using a manual search task similar to the one used by Santos et al. (2002). Monkeys saw the Experimenter (E) taking a white piece of (fake) fruit out of the inner part of a fruit, e.g., coconut and placing it inside the (pre-baited) box. In the consistent condition, the monkeys then really found a piece of coconut. In the inconsistent condition, in contrast, the monkey found a similarly looking piece of apple. Subjects searched longer in the inconsistent condition than during the consistent one.

In the present studies, we followed up on these findings, extending them to great apes. Furthermore, a refined procedure was used which takes into account potential limitations in the previous studies. Firstly, in the Santos et al. (2002) and Phillips and Santos (2007) studies the dependent search measure was defined differently than in the infant studies and in a way that makes the findings difficult to interpret: as searching in or around the box. This seems a less than straightforward measure for the monkeys' abilities to track how many and what kinds of objects are in the box. ${ }^{4}$ In the current studies, therefore we added a more stringent measure of manually searching inside the box only.

Secondly, we added a control phase as it was used in infant studies. Particularly, in the study by Van de Walle et al. (2000), in the unexpected conditions, there were several phases: In the first phase, after children found one object, they were allowed to search the box (which was - unbeknownst to them - empty) for the second object they expected for a given amount of time. Then, the expected object was surreptitiously put back into the box and the infants found it, and in a second control phase the infants were then allowed to search further. The logic was the following: If the infants in fact had the determinate expectation to find a specific object, they should then quit searching after they found it. This was indeed the pattern that was found with 12-month-old infants. Such a control phase was missing from the studies by Santos et al. (2002) and Phillips and Santos (2007). Perhaps the monkeys would have continued searching in and around the box even after having found the expected object, simply due to increased arousal. We do not think this is a likely possibility, but in the absence of the required control phase it cannot be ruled out.

In sum, kind-based object individuation emerges in human ontogeny at latest towards the end of the first year of life (and possibly earlier), and has been argued

\footnotetext{
${ }^{4}$ In fact, Santos et al. (2002) are well aware of this methodological constraint of their search measure: "Our measure of searching includes the time an animal spends searching for missing objects, as well as the time subjects spend looking in response to various violations [...]. These two measures are confounded in our searching measure in a way that they are not in the infancy measures [...]. It may well turn out that other methods yield more subtle differences across conditions, particularly a method that distinguishes between searching in the box and looking at the box" (p. 261). The purpose of our current studies is to devise such an improved and more subtle measure.
} 
to be linguistically determined and therefore uniquely human. Existing studies in this area with non-human primates present prima facie evidence against this latter claim, but remain somewhat inconclusive due to methodological limitations. In the present studies, therefore, we set out to test this claim and follow up on existing studies with a refined methodology with great apes.

\section{Experiment 1: Spatiotemporal information}

\subsection{Methods}

\subsubsection{Participants}

Fourteen chimpanzees (Pan troglodytes), five bonobos (Pan paniscus) and four gorillas (Gorilla gorilla) participated in the present study. There were 7 males and 16 females ranging in age between 5 and 28 years ( $M=15$ years and 5 months). All subjects had previously participated in a variety of cognitive studies (see Table 1 for rearing and testing history). All subjects were socially housed at the Wolfgang Köhler Primate Research Center, Leipzig Zoo, Germany. All indoor and outdoor enclosures were furnished with vegetation, climbing structures and visual barriers. Subjects were neither food- or water-deprived during the experiment.

\subsubsection{Apparatus}

We used an opaque plastic box $(40 \times 40 \times 34.5 \mathrm{~cm}$, see Fig. 1) with a circular opening (approx. 8.5 in diameter) drilled on its top middle part through which the experimenter (E) could insert her hand and forearm to add rewards (and pretend to take out rewards; see below). The box had a false roof $(9 \mathrm{~cm}$ high from its top part), not visible for the subjects, where a reward could be surreptitiously stored. The frontal part of the box (subjects' point of view) had a Plexiglas sliding door, which once opened by E, would allow subjects to reach inside the box. The front opening of the box ( $13 \mathrm{~cm}$ wide $\times 6 \mathrm{~cm}$ high) was covered with a curtain made of burlap bag material to prevent subjects to look inside before and while reaching. In a research station the box was attached to a clear Plexiglas panel which had a small circular opening on its left part (from E's point of view).

\subsubsection{Procedure}

Testing was done by one $\mathrm{E}$ who was assisted by a cameraperson whose role besides operating the camera was to time the trials and inform $E$ when the subject had retrieved the reward. There were two phases in the experiment.

Familiarization phase. Before the test phase all subjects went through a familiarization phase with the apparatus in which they received two kinds of trials sequentially presented: (1) one-object familiarization trial, in which the subject saw E placing one reward on top of the experimental box, followed by its introduction inside it (through the opening on its top part). After the hiding process, the frontal sliding door of the box was opened and the subject was allowed to reach. As soon as the reward had been found, the door was immediately closed; (2) two-object familiariza- 
Table 1

Age, sex, rearing history, and test history of the subjects that participated in the studies

\begin{tabular}{|c|c|c|c|c|c|}
\hline Species & Subject & $\begin{array}{l}\text { Age } \\
\text { (years, months) }\end{array}$ & Sex & $\begin{array}{l}\text { Rearing } \\
\text { history }\end{array}$ & $\begin{array}{l}\text { Test history } \\
\text { (participation in } \\
\text { Experiments } 1 \text { and/or 2) }\end{array}$ \\
\hline \multirow[t]{5}{*}{ Bonobo } & Joey & 21.5 & M & Hand reared & 1,2 \\
\hline & Kuno & 7.5 & M & Hand reared & 1,2 \\
\hline & Limbuko & 8.5 & $\mathrm{M}$ & Hand reared & 1,2 \\
\hline & Ulindi & 10.5 & $\mathrm{~F}$ & Mother & 1,2 \\
\hline & Yasa & 7.5 & $\mathrm{~F}$ & Mother & 1,2 \\
\hline \multirow[t]{16}{*}{ Chimpanzee } & Alex & 4.0 & $\mathrm{M}$ & Hand reared & 2 \\
\hline & Alexandra & 5.5 & $\mathrm{~F}$ & Hand reared & 2 \\
\hline & Brent & 5.0 & M & Mother & 1,2 \\
\hline & Corry & 27.5 & $\mathrm{~F}$ & Hand reared & 1,2 \\
\hline & Dorien & 23.5 & $\mathrm{~F}$ & Hand reared & 1,2 \\
\hline & Fifi & 11.5 & $\mathrm{~F}$ & Mother & 1,2 \\
\hline & Fraukje & 28.5 & $\mathrm{~F}$ & Hand reared & 1,2 \\
\hline & Frodo & 10.5 & $\mathrm{M}$ & Mother & 1,2 \\
\hline & Gertrudia & 11.5 & $\mathrm{~F}$ & Mother & 1,2 \\
\hline & Jahaga & 11.5 & $\mathrm{~F}$ & Mother & 1,2 \\
\hline & Natascha & 24.5 & $\mathrm{~F}$ & Hand reared & 1,2 \\
\hline & Patrick & 7.5 & M & Mother & 1,2 \\
\hline & Pia & 5.0 & $\mathrm{~F}$ & Mother & 1,2 \\
\hline & Riet & 27.0 & $\mathrm{~F}$ & Hand reared & 1,2 \\
\hline & Robert & 28.5 & M & Hand reared & 1,2 \\
\hline & Sandra & 11.5 & $\mathrm{~F}$ & Mother & 1 \\
\hline \multirow[t]{4}{*}{ Gorilla } & Bebe & 25.5 & $\mathrm{~F}$ & Mother/hand reared & 1,2 \\
\hline & N'Diki & 27.5 & $\mathrm{~F}$ & Mother/hand reared & 1,2 \\
\hline & Ruby & 6.5 & $\mathrm{~F}$ & Hand reared & 1,2 \\
\hline & Viringika & 9.5 & $\mathrm{~F}$ & Mother & 1,2 \\
\hline
\end{tabular}

Note. F, female; $\mathrm{M}$, male.

tion trial, in which the subject saw E placing two identical rewards on top of the experimental box (one on each side of the box), and then introducing them, one by one (through the opening on its top part). Immediately after the hiding process, the sliding door was opened and the subject was allowed to reach. As soon as the rewards had been found, the sliding door was immediately closed.

Only after subjects had successfully passed the familiarization phase, test trials were administered. The criterion for advancing a subject to the next phase consisted of succeeding in retrieving the rewards in both kinds of familiarization trials. If a subject got the reward in the one-object familiarization trial, but did not get both of them in the two-object familiarization trial, both trials were redone until the subject got the rewards in both trials in sequence.

Test phase. The objects used in this phase were always grapes (as similar as possible in their properties, i.e. color, size and shape). There were two conditions (see Fig. 2 for a schematic illustration of the whole procedure). In the one-object condition the subject saw E placing one grape on top of the experimental box followed by 


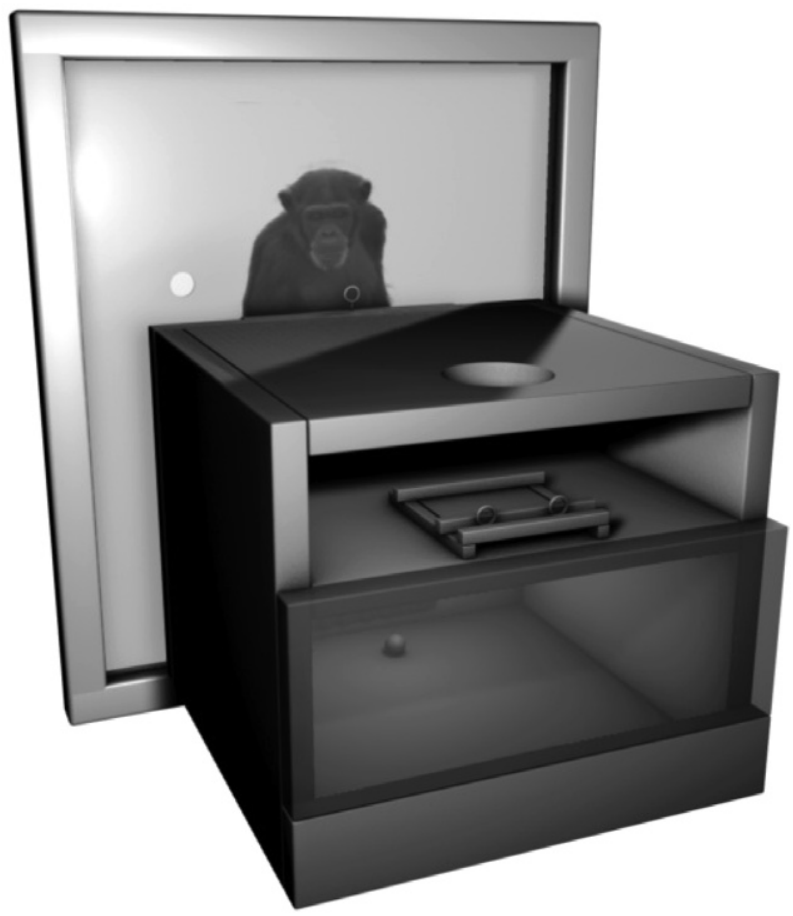

Fig. 1. Drawing of the experimental apparatus used in Experiment 1 (E's perspective). The X-ray view allows the reader to see the location of the grape inside the box.

its introduction inside the box (through the opening in its top part). After the hiding process was completed, the sliding door was opened and the subject was allowed to reach inside the box to get the reward. As soon as the grape had been found and the subject had retracted her hand, the door was immediately closed. Then, E re-opened the door for a 20 -s reach period $\left(\mathrm{RP}_{1}\right)$. After this period was over, the door was closed and the trial was over.

In the two-object condition, the subject saw E placing two identical grapes on the top of the experimental box (one on each side of the box) followed by their introduction one by one inside the box. However, the second grape was always placed surreptitiously on the false roof so that the subject could only access one grape. After the hiding process was completed, the sliding door was opened and the subject was allowed to reach inside the box to get the reward. As soon as the grape in the box had been found, the door was immediately closed and re-opened for a first 20 -s reach period $\left(\mathrm{RP}_{1}\right)$. After this reach period was over, the door was closed again, E inserted her hand and forearm inside the box, pretended to reach for the "missing" grape, took it from the false roof and gave it to the subject through a small hole on the Plexiglas panel (to which the box was attached). As soon as the subject received the grape from E's hand, the sliding door was re-opened one last time for a second 20-s reach period $\left(\mathrm{RP}_{2}\right)$. After this period had elapsed, the door was closed and the trial was over. 

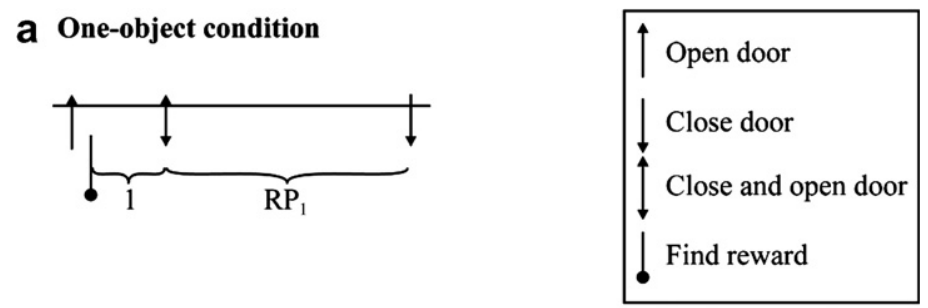

b Two-object condition

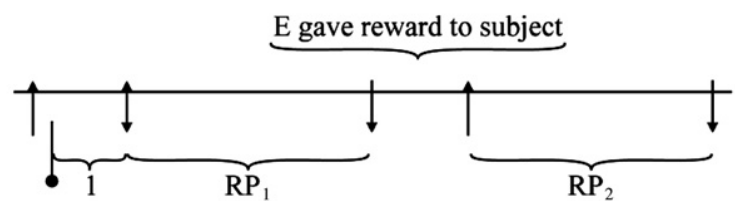

Fig. 2. Illustration of the procedure during one and two-object conditions. Number 1 indicates the period of re-reaching immediately after the subject had found the reward and before the experimenter had time to close the sliding door of the box.

On numerous occasions, subjects re-reached inside the box immediately after finding the first grape, and before $\mathrm{E}$ had a chance to close the sliding door. E then waited until the subject withdrew its hand to close the sliding door and continue with the test as described above. Each subject received two test trials per condition. The order of trial presentation was alternated between trials of each condition, with half of the subjects receiving a one-object test trial first, the other half receiving a two-object test trial first. In both kinds of conditions, visual contact was only established during the hiding process of the grapes and when E pretended to take the "missing" grape out of the box (in the two-object condition).

\subsubsection{Predictions}

Following Van de Walle et al. (2000), we predicted that if great apes were capable of individuating "Spelke objects" according to spatiotemporal information, they should reach more in the first 20 -s reach period $\left(\mathrm{RP}_{1}\right)$ for the two-object condition compared to the one-object condition because they would expect a second object in the former but not in the latter. In addition, reach effort should decrease in the second 20-s reach period $\left(\mathrm{RP}_{2}\right)$ compared to $\mathrm{RP}_{1}$ in the two-object condition because subjects had received the expected number of objects before the $\mathrm{RP}_{2}$ started. Due to the directional nature of our predictions, statistical tests were one-tailed unless noted otherwise.

\subsubsection{Coding and data analysis}

An observer coded all the digitalized videos using the Interact ${ }^{\circledR}$ software (version 7). The main dependent measure was reaching into the box, which was operationally defined as introducing the hand inside the box at least up to the second hand knuckles. Both reaching frequency and reaching duration were considered. For each sub- 
ject, the average score over the two trials in each condition were computed for frequency and duration, yielding one frequency and one duration score for the oneobject condition, and two frequency and two duration scores for the two-object condition (for $\mathrm{RP}_{1}$ and $\mathrm{RP}_{2}$ ). Analogous scores were computed for re-reaching because this measure seemed to indicate in the most direct way whether subjects expected to find additional rewards in the box.

As the data failed to fulfill some requirements for parametric statistics, all analyses were conducted with non-parametric tests (Kruskall-Wallis, Wilcoxon signed rank test, exact Wilcoxon test for $N \leqslant 15$ ). First, some control analyses to test for species and age effect were run subjects were split into four age groups: infant (until 5 years old), juvenile (from 5 up to 8 years old), sub-adult (from 8 up to 11 years old), and adult (from 11 years old on). As the average frequency and duration of reaching were uninformative to test for species or age effects, we used scores based on the differences between (a) the one-object condition and the two-object condition for $\mathrm{RP}_{1}$, (b) the one-object condition and the two-object condition for immediately re-reaching, and (c) $\mathrm{RP}_{1}$ and $\mathrm{RP}_{2}$ within the two-object condition.

The main analyses tested the effect of condition (one-object vs. two-object) on the average frequency and duration of reaching during the designated 20 -s reach periods. An analogous analysis was conducted for re-reaching. To assess inter-observer reliability, a second observer coded a random sample of $20 \%$ of the trials. Inter-observer reliability was high for frequency (Pearson correlation $r=.965, P<.001, N=50$ and weighted $K=.977$ ) and for duration (Pearson correlation $r=.970, P<.001$, $N=50)$.

\subsection{Results}

No species or age groups' effects were found in any of the difference scores above mentioned both for reach frequency and duration (Kruskal-Wallis, $p>.05$ ).

Fig. 3 depicts the average frequency and duration of reaching during the reach periods in each condition. Focusing on the first reaching period $\left(\mathrm{RP}_{1}\right)$, subjects reached significantly more often in the two-object compared to the one-object condition (Wilcoxon test: $Z=2.84, N=18, P=.002$ ). Similarly, in $\mathrm{RP}_{1}$ subjects reached longer in the two-object compared to the one-object condition (Wilcoxon test: $Z=2.97, N=21, P=.002$ ). Focusing on the two-object condition and comparing the first and the second reach periods, subjects reached significantly longer during $\mathrm{RP}_{1}$ than during $\mathrm{RP}_{2}$ (Wilcoxon test: $Z=1.79, N=23, p=.04$ ). In contrast, there were no significant differences between periods for reaching frequency (Wilcoxon test: $Z=1.134, N=22, P=.13$ ).

Fig. 4 depicts the average frequency and duration of re-reaching inside the box immediately after having found the first grape in each condition. Subjects re-reached significantly more often (Wilcoxon test: $T=2.16, N=11, P=.014$ ) and longer ( $\mathrm{Wil}$ coxon test: $Z=1.73, N=17, P=.042$ ) in the two-object condition compared to the one-object condition. 

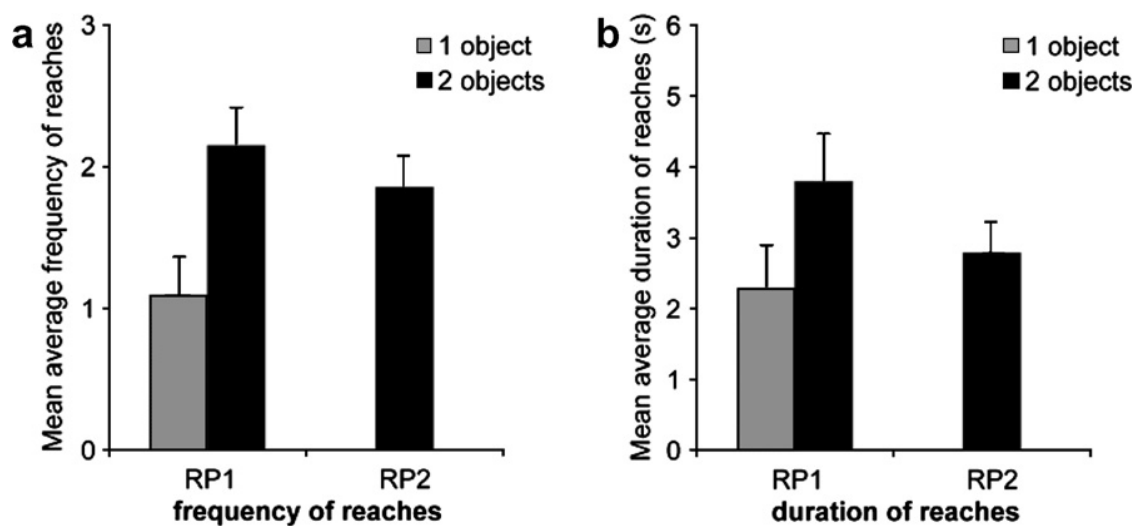

Fig. 3. Average $(+S E)$ frequency (a) and duration (b) of reaches during the 20 -s periods $\left(\mathrm{RP}_{1}\right.$ and $\left.\mathrm{RP}_{2}\right)$ in both kinds of conditions.
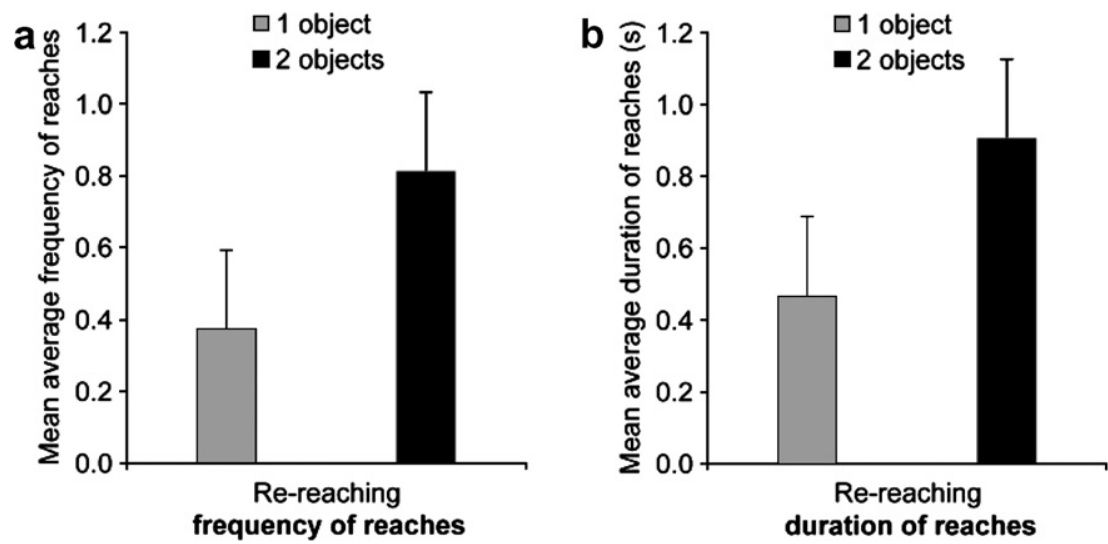

Fig. 4. Average $(+S E)$ frequency (a) and duration (b) of re-reaching inside the box immediately after having found the first grape, on both kinds of conditions.

\subsection{Discussion}

Subjects reached significantly more often and longer in the two-object compared to the one-object condition. Similarly, subjects also re-reached more often and for a longer time after having found the first grape in the two-object condition compared to the one-object condition. Although subjects also reached for a longer time in the first reach period compared to the second one in the two-object condition, they did not reach significantly more frequently. It is not clear why these two measures diverge at this point. However, it should be noted that duration of reaching seems more informative as a measure of search behavior than frequency of reaches (compare reaching once for $10 \mathrm{~s}$ to reaching five times for $1 \mathrm{~s}$ each), and it is also the measure that has been used in the previous studies with non-human primate studies which we followed up on (see Phillips and Santos, 2007; Santos et al., 2002). 
In sum, the results of the present study not only replicate existing findings from other manual search studies with human and non-human primates (Santos et al., 2002; Van de Walle et al., 2000, respectively) but also extend some of those findings for non-human primates.

First, the more stringent dependent measure used here (reaching inside the box as opposed to reaching around the box) allows us to make a stronger claim that subjects expected to see certain number of objects inside the box. Second, the inclusion of the immediate re-reaching measure represents an improvement over other studies because it reduces performance factors such as memory load and attention compared to delayed reach periods. Third, following Van de Walle et al. (2000) we included a second reach period $\left(\mathrm{RP}_{2}\right)$ in the two-object condition that permitted us to rule out simple reach strategies that are not based on object individuation. For instance, subjects may have followed the following strategy: "The more stuff you see, the longer you search". Consistent with an object individuation account, subjects reached longer in the first 20-s reach period $\left(\mathrm{RP}_{1}\right)$ compared to the second 20-s reach period $\left(\mathrm{RP}_{2}\right)$ within two-object condition.

\section{Experiment 2: Property/kind information}

The previous experiment showed that subjects used spatiotemporal information to individuate objects. The current experiment was designed to test whether great apes could use a more sophisticated source of information, the property/kind information of objects. We improved the design of the current experiment in two ways. First, both conditions, not just the two-object condition, had a second reach period $\left(\mathrm{RP}_{2}\right)$. Second, the subjects were allowed to retrieve the "missing" reward from inside the box themselves (rather than being handed by $\mathrm{E}$ ) once the door was reopened after $\mathrm{RP}_{1}$.

\subsection{Methods}

\subsubsection{Participants}

The same subjects as in Experiment 1 were tested, with the exception of one chimpanzee (Sandra) who did not cooperate. Two additional chimpanzees (Alex and Alexandra), who had not been tested in Experiment 1, participated in the current one. Therefore, fifteen chimpanzees (Pan troglodytes), five bonobos (Pan paniscus) and four gorillas (Gorilla gorilla) participated in the present study (see Table 1). There were 8 males and 16 females ranging in age between 4 and 28 years ( $M=14$ years and 8 months).

\subsubsection{Apparatus}

The same apparatus as in Experiment 1 was used with the following improvements. First, a horizontal sliding door (trap) was constructed on one of the sides of the false roof of the box to facilitate the surreptitious introduction of a reward. Second, sliding corners were added inside the box to prevent the "missing" reward, 
dropped with the help of the horizontal sliding door, from getting obstructed on them.

\subsubsection{Procedure}

Testing was done by the same $\mathrm{E}$ as in Experiment 1 who was assisted by a cameraperson. Both experimenters followed the same basic procedure as in Experiment 1. There were two phases in the experiment.

Familiarization phase. Only subjects that had not participated in the previous experiment (i.e. Alex and Alexandra) received this phase that consisted of the same procedure as in Experiment 1.

Test phase. Six kinds of food items (as dissimilar as possible in kind and properties) were used as rewards, two in each unexpected trial (e.g., half a nut and a banana slice), and one in each expected trial (e.g., a piece of bread). There were two conditions (see Fig. 5 for a schematic illustration of the whole procedure). In the expected condition subjects saw $\mathrm{E}$ introducing reward $\mathrm{A}$ inside the box and when allowed to reach they found reward $A$. In the unexpected condition the box was initially baited with reward B (subjects were unaware of this manipulation) and they saw E introducing reward A. However, reward A was surreptitiously stored on the horizontal sliding door of the false roof. Once the sliding door was opened, subjects found reward $\mathrm{B}$, different in properties and kind from the one they saw being hidden.

In both conditions, after the subjects had found the reward, the sliding door was immediately closed (again, if the subject immediately re-reached, the same procedure as in Experiment 1 was followed) and re-opened for a 20 -s period ( $\left.\mathrm{RP}_{1}\right)$. After that reach period was over, the sliding door was closed and the horizontal sliding door was simultaneously opened so that the "missing" reward (in the unexpected condition) fell near the front corners of the box. This created the impression that the reward had been there from the beginning of the trial and that subjects had not found it before because of its difficult location, not because $E$ had manipulated it. While E closed and opened the sliding door, simultaneously with the opening of
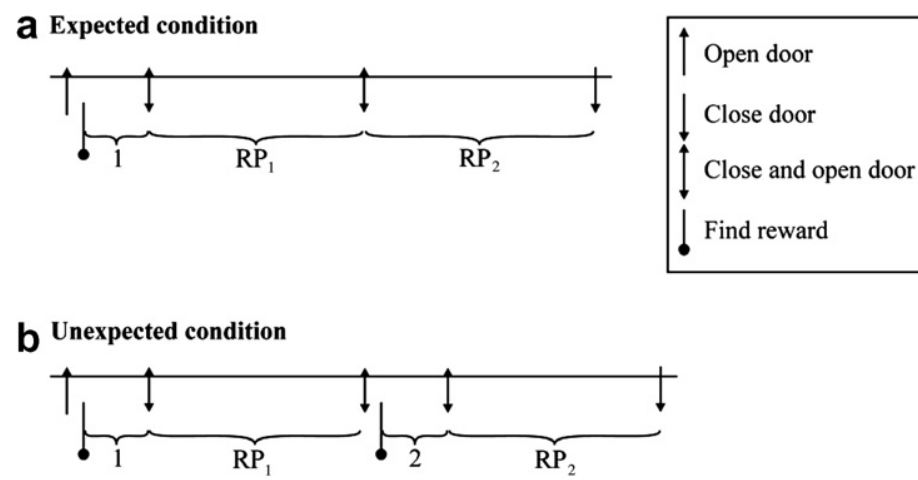

Fig. 5. Illustration of the procedure during expected and unexpected conditions. Numbers 1 and 2 indicate the periods of re-reaching immediately after the subject had found the reward and before the experimenter had time to close the sliding door of the box. 
the trap, she spoke loudly (i.e. "Look at that!") to prevent subjects from using auditory cues that may have emanated from the fall of the reward (applied in both conditions). If subjects did not reach inside the box, or if they failed to find the "missing" reward in the unexpected condition, the trial was ended after $20 \mathrm{~s}$. If subjects retrieved the "missing" reward the door was immediately closed and re-opened again for a last 20 -s reach period $\left(\mathrm{RP}_{2}\right)$.

Each subject received two trials per condition, except two subjects who only received one trial each per condition due to fussiness. The order of presentation alternated between trials of each condition, with half of the subjects receiving an expected trial first, the other half receiving an unexpected trial first.

\subsubsection{Predictions}

We predicted that if great apes were capable of object individuation according to property/kind information, they should reach significantly more often and longer in the first reach period $\left(\mathrm{RP}_{1}\right)$ of the unexpected condition than that of the expected condition because they would expect a different object than the one they found in the former but not in the latter. In addition, reach effort should decrease in the second reach period $\left(\mathrm{RP}_{2}\right)$ compared to the first reach period $\left(\mathrm{RP}_{1}\right)$ after finding the "missing" reward in the unexpected condition. Again, due to the directional nature of the predictions, one-tailed statistics were applied.

\subsubsection{Coding and data analysis}

Data coding, dependent measures and analyses were done in the same way as in Experiment 1 . However, the analysis of the second reach periods was complicated by the fact that only 12 subjects retrieved the "missing" reward in the unexpected condition (out of the 12 subjects who never retrieved the "missing" reward, eight subjects did reach but could not find it, and only four never reached at all). Therefore, only those trials in which subjects recovered the missing reward were analyzed. Six subjects found the reward in both trials and six subjects only found it in one trial. Average scores were then computed for each subject only over those trials in which it found the "missing" reward.

To test for effects of species and age, analogous analyses over difference scores were run as in Experiment 1. The following main analyses were conducted. First, we compared the first 20 -s reach period $\left(\mathrm{RP}_{1}\right)$ across conditions. Second, we compared the performance of the subjects who found the "missing" reward in at least one of the two unexpected trials across all reach periods. This analysis allowed us to see whether upon finding the "missing" reward in the unexpected condition, subjects decreased their reach effort in subsequent periods, and to compare their performance with the condition in which they found the expected reward. Unlike the previous analysis, this analysis was based on a sub-sample of 12 subjects. The third main analysis investigated the performance of the subjects during the re-reaching periods immediately after having found the reward. Again, the analysis of the second re-reaching period belonging to the unexpected condition was conducted on only those subjects who found the "missing" reward in at least one of the two unexpected trials. 
Inter-observer reliability was high both for frequency (Pearson correlation $r=.989, P<.001, N=60$; and weighted $K=.978$ ) and duration measures (Pearson correlation $r=.982, P<.001, N=60$ ).

\subsection{Results}

No species or age group effects were found in any of the difference scores between the different reach periods both for reaching frequency and duration (Kruskal-Wallis, $p>.05)$.

Fig. 6 depicts the average frequency and duration of reaching and re-reaching during the first reach period in each condition $\left(\mathrm{RP}_{1}\right)$. In $\mathrm{RP}_{1}$, subjects reached significantly more often (Wilcoxon test: $T=2.69, N=15, P=.003$ ) and for a longer time (Wilcoxon test: $Z=2.43, N=21, P=.008$ ) in the unexpected compared to the expected condition. This result is confirmed by the re-reaching data. Subjects rereached significantly more often (Wilcoxon test: $T=1.73, N=10, P=.055$ ) and longer in the unexpected compared to the expected condition (Wilcoxon test: $T=1.89, N=13, P=.03$ ).

Fig. 7 depicts the average frequency and duration of reaching during both reach periods in each condition for those 12 subjects who found the "missing" reward in at least one trial of the unexpected condition. Focusing on the unexpected condition, subjects reached significantly more often (Wilcoxon test: $T=3.05, \quad N=12$, $P=.000)$ and for a longer time (Wilcoxon test: $T=2.35, N=12, P=.008$ ) in the first compared to the second reach period. In contrast, subjects in the expected condition did not reach differentially in the first compared to the second reach periods for frequency (Wilcoxon test: $T=.64, N=8, P=.594$, two-tailed) or duration ( $\mathrm{Wil}$ coxon test: $T=1.09, N=12, P=.301$, two-tailed)

Fig. 8 depicts the average frequency and duration of re-reaching during both rereaching periods in each condition for those 12 subjects who found the "missing"
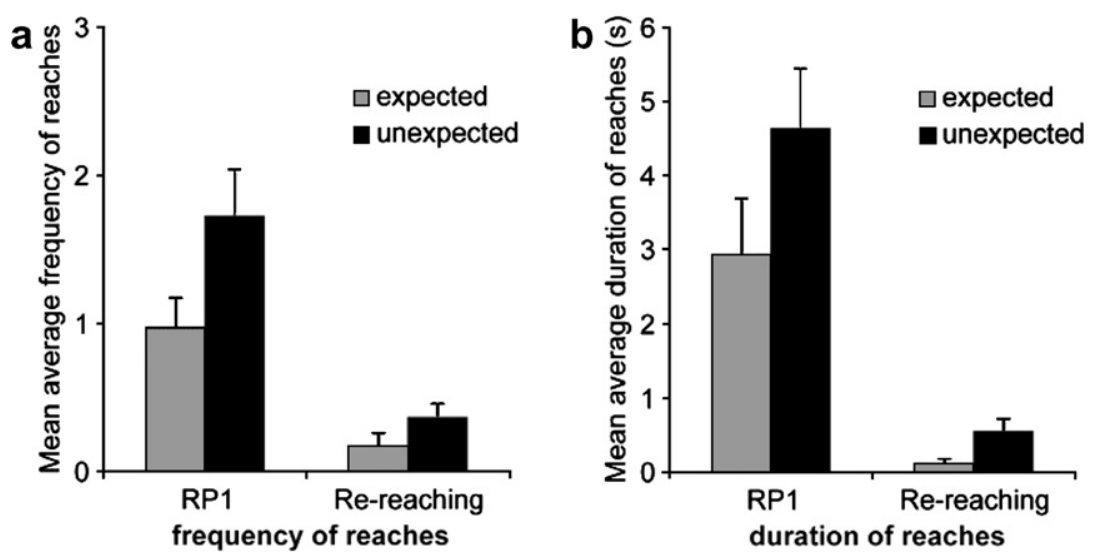

Fig. 6. Average $(+S E)$ frequency (a) and duration (b) of reaches during the first 20 -s period $\left(\mathrm{RP}_{1}\right)$ as well as during the first re-reaching period in both conditions. 

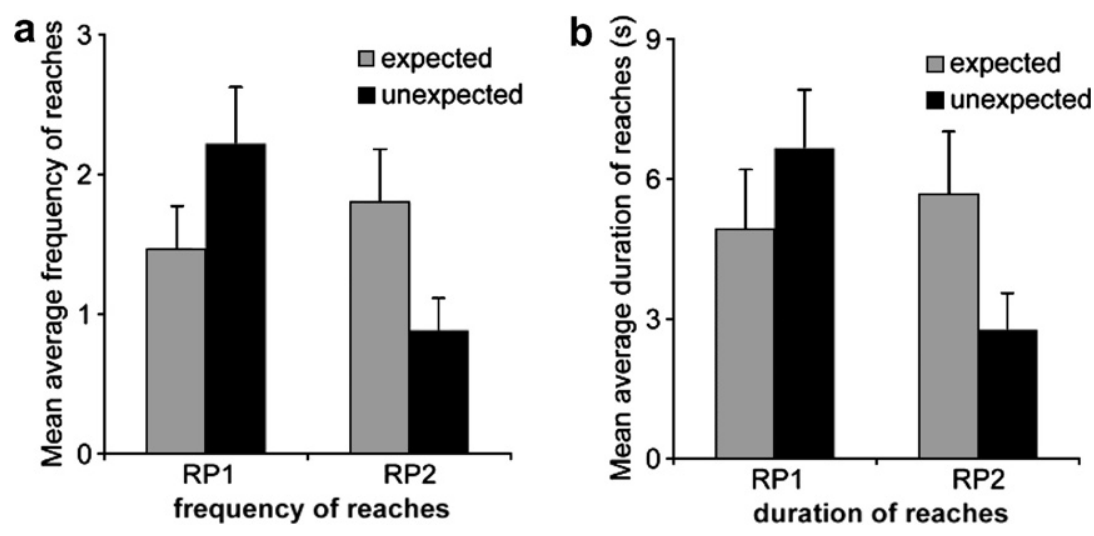

Fig. 7. Average $(+S E)$ frequency (a) and duration (b) of reaches during both 20 -s periods $\left(\mathrm{RP}_{1}\right.$ and $\left.\mathrm{RP}_{2}\right)$ of those subjects $(N=12)$ who found the "missing" reward in at least one trial of the unexpected condition.
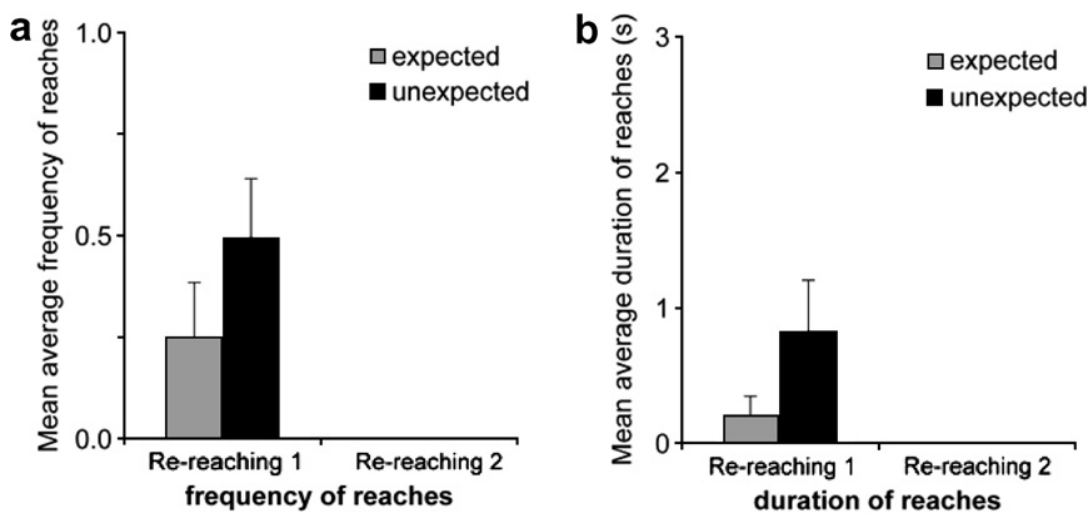

Fig. 8. Average $(+S E)$ frequency (a) and duration (b) of both re-reaching periods of those subjects $(N=12)$ who found the "missing" reward in at least one trial of the unexpected condition.

reward in at least one trial of the unexpected condition. Data on re-reaching frequency and duration reinforced the results obtained in the unexpected condition (see Fig. 7). Subjects re-reached significantly more often (Wilcoxon test: $T=2.46$, $N=7, P=.008$ ) and for longer durations (Wilcoxon test: $T=2.367, N=7$, $P=.008$ ) upon finding an unexpected reward (period before $\mathrm{RP}_{1}$ ) as opposed to the "missing" reward (period before $\mathrm{RP}_{2}$ ). In fact, none of the 12 subjects who found the "missing" reward in the unexpected condition, re-reached immediately after finding this reward.

\subsection{Discussion}

Subjects reached significantly more often and longer in the first reach period $\left(\mathrm{RP}_{1}\right)$ of the unexpected condition compared to the expected condition. Furthermore, upon 
finding the "missing" reward in the unexpected condition, subjects decreased their search effort. ${ }^{5}$ Again, these two main results were reinforced by the re-reaching data: Great apes re-reached more often and longer after having found the first reward in the unexpected than in the expected condition. In the unexpected condition, the subjects never re-reached after having found the second, expected reward. Thus, the results across the different reaching measures suggest that subjects are capable of object individuation based on the processing of property/kind information.

As the findings of Experiment 1, the current results replicate and extend those found in human and non-human primates (Santos et al., 2002; Uller et al., 1997; Van de Walle et al., 2000). Even with the more stringent search measure used here (i.e. reaching in the box only) and with the second reach periods (controlling for simpler search strategies), the great apes' search behavior clearly suggests that they individuated objects according to their properties/kind.

\section{General discussion}

The present results corroborate and extend the findings of existing studies on object individuation in non-human primates (Phillips and Santos, 2007; Santos et al., 2002; Uller et al., 1997). Using a more stringent methodology than that applied in previous studies revealed that great apes (bonobos, chimpanzees and gorillas) individuated food objects based both on spatiotemporal information of two objects presented simultaneously (Experiment 1), as well as on property/kind information (Experiment 2). In fact, the great apes in both experiments performed as human infants from 10 to 12 months of age do in analogous manual search tasks (Van de Walle et al., 2000; Xu \& Baker, 2005).

These findings clearly suggest that non-human primates are capable of some sophisticated form of individuating objects beyond mere spatiotemporal tracking of "Spelke objects". 6 This more sophisticated form of object individuation emerges in human ontogeny (at latest) at around one year of age and that has been found to correlate with language comprehension.

Now, what does this mean for the relation of language and thought in object cognition? If one takes the property/kind condition to measure object individuation according to object kinds and to require sortal concepts (as is mostly done in the

\footnotetext{
${ }^{5}$ Surprisingly, in contrast to the unexpected condition, reaches in the expected condition did not decrease during the second reach period $\left(\mathrm{RP}_{2}\right)$. One possible explanation for this surprising result might be that subjects in the unexpected condition simply have already searched longer than in the expected condition (thus have become tired). They have searched for $20 \mathrm{~s}\left(\mathrm{RP}_{1}\right)$, then searched again to find the hidden food, and then came $\mathrm{RP}_{2}$. A complementary possibility is this: Subjects in the expected condition found the reward before $\mathrm{RP}_{1}$ already, and therefore, afterwards (during $\mathrm{RP}_{1}$ and $\mathrm{RP}_{2}$ ) there is barely anything else to do for them, so why not just fill in the time in both reaching periods in the same manner?

${ }^{6}$ One interesting question is how domain-general these forms of object individuation are, particularly outside the domain of food. As all previous studies on object individuation in non-human primates, only food items were used in the present studies. This is due to practical reasons - it is very difficult to keep the subjects motivated without using food.
} 
infant literature since Xu and Carey's (1996) pioneer study), then the claim that language is necessary for kind-based object individuation seems to be refuted by the converging evidence of previous and the present findings with non-human primates.

However, both Uller et al. (1997) and Xu (2002) have argued that more parsimonious analyses of property/kind conditions used with non-human primates are possible. Above all, subjects might merely focus on the properties that one object has and that another one misses, without necessarily conceiving of the two objects as objects of different kinds. For example, on seeing a carrot at time 1 the subject registers "longness" and "orangeness". At time 2, when she finds a banana, she registers "longness" and "yellowness". And as, so to speak, some "orangeness" is missing, from her point of view, she continues searching. In other words, sophisticated feature-placing might be sufficient for mastering property/kind conditions as they were used with non-human primates in Uller et al. (1997), Santos et al. (2002) and in the present experiments.

It should be noted, though, that these concerns raised with regard to studies with non-human primates equally apply to infant studies on object individuation. Both in the habituation studies (Xu \& Carey, 1996 and follow-ups) and in the manual search studies (Van de Walle et al., 2000; Xu \& Baker, 2005), the same confound between kind membership and properties was present. Thus, the pre-linguistic infants who succeeded in the property/kind conditions might have relied on property differences rather than on kind differences in the same way as the non-human primates.

Behind this methodological problem lies a deeper theoretical problem: Property differences and kind differences are usually confounded. Crucially, however, there are cases where differences/changes of properties and differences/changes of kind can be teased apart. In particular, non-essential (superficial) properties of an object can change while it still remains of the same kind. For example, a raccoon that gets shaved and painted like a skunk remains the same raccoon despite quite drastic property changes, to take an example from studies with older children (e.g., Keil, 1989). In such studies verbal judgments (that the raccoon remained the same raccoon) are taken as indicative of children's understanding of kind constancy in the face of superficial property transformations.

While such a logic cannot be directly applied in non-linguistic tasks, one line of research with infants is noteworthy in this context: Xu, Carey, and Quint (2004), in a habituation study, found that infants in some cases disregard (superficial) property differences (e.g., color, shape, size) between members of one kind though they take into account similar property differences when they are between members of different kinds.

For example, infants saw a red ball disappear behind an occluder (time 1), then saw a green ball appear from behind the occluder and disappear again (time 2). They then either found two balls (expected) or only one ball (unexpected). Remarkably, 12-month-olds in this study did not look longer at the unexpected versus the expected events (see Feigenson \& Carey, 2003, for similar results regarding size differences with a manual search methodology).

The interpretation goes as follows: if infants only track properties they should in both conditions look for the missing properties after having found only one object, 
but be satisfied with having found all properties after having found two objects. In contrast, if infants track objects according to their kinds, they might just register "a ball" a time 1, and "a ball" at time 2 , without tracking their property differences and realizing that they were numerically distinct balls, they should be satisfied after having found one ball.

These results do of course not directly show that infants used sortal concepts in these studies. But taken together with the property/kind conditions of the habituation and manual search studies, they can be seen as converging evidence making it plausible that human infants use kind-based object individuation beyond mere property tracking (feature placing) from (at latest) around 1 year.

In the case of non-human primates, the present experiments add to the growing body of evidence that non-linguistic animals are capable of individuating objects according to their properties/kind. Whether this reflects an ability to apply sortal kind concepts, or rather only a simpler form of property tracking, awaits further empirical clarification. Particularly, it is an open question for future research whether a similar pattern of disregarding property differences between objects of the same kind as in the Xu et al. (2004) and Feigenson and Carey (2003) studies can be found. As in the case of human infants, such a finding would add to the plausibility of the claim that non-human primates are capable of true kind-based object individuation.

\section{Acknowledgements}

This research was partially supported by a Ph.D. grant from the "Fundação para a Ciência e a Tecnologia", Portugal to Natacha Mendes. We are very grateful to Heinz Gretscher and Sebastian Tempelmann for their help during the data collection. We also thank Daniel Stahl for statistical help, and Knut Finstermeier for the elaboration of the apparatus' figure as well as the quality of the remaining ones. Thank you to Jens Günther for the construction of the experimental apparatus, and Raik Pieszek, Christel Schneider and Martina Neumann for logistical help.

\section{References}

Baillargeon, R. (1987). Young infants' reasoning about the physical and spatial properties of a hidden object. Cognitive Development, 2, 375-392.

Bonatti, L., Frot, E., Zangl, R., \& Mehler, J. (2002). The human first hypothesis: Identification of conspecifics and individuation of objects in the young infant. Cognitive Psychology, 44(4), 388-426.

Clark, A. (2004). Feature-placing and proto-objects. Philosophical Psychology, 17(4), 443-469.

Davidson, D. (1982). Rational Animals. Dialectica, 36, 317-327.

Dummett, M. (1993). The origins of analytical philosophy. London: Duckworth.

Evans, G. (1982). The varieties of reference. Oxford: Oxford University Press.

Feigenson, L., \& Carey, S. (2003). Tracking individuals via object-files: Evidence from infants' manual search. Developmental Science, 6(5), 568-584.

Keil, F. (1989). Concepts, kinds and cognitive development. Cambridge, MA: MIT Press. 
Needham, A., \& Baillargeon, R. (2000). Infants' use of featural and experiential information in segregating and individuating objects: A reply to Xu, Carey and Welch (2000). Cognition, 74(3), 255-284.

Phillips, W., \& Santos, L. (2007). Evidence of kind representations in the absence of language: Experiments with rhesus monkeys (Macaca mulatta). Cognition, 102, 455-463.

Pylyshyn, Z. (2000). Situating vision in the world. Trends in Cognitive Science, 4(5), 197-207.

Quine, W. V. O. (1960). Word and object. Cambridge, MA: MIT Press.

Quine, W. V. O. (1981). Theories and things. Cambridge: Harvard University Press.

Santos, L. R., Sulkowski, G. M., Spaepen, G. M., \& Hauser, M. D. (2002). Object individuation using property/kind information in rhesus macaques (Macaca mulatta). Cognition, 83(3), 241-264.

Sellars, W. (1963). Empiricism and the philosophy of mind. In W. Sellars (Ed.), Science, perception and reality. London: Routledge \& Kegan Paul.

Spelke, E. S. (1990). Principles of object perception. Cognitive Science, 14, 29-56.

Spelke, E. S., Kestenbaum, R., Simons, D., \& Wein, D. (1995). Spatio-temporal continuity, smoothness of motion and object identity in infancy. British Journal of Developmental Psychology, 13, 113-142.

Strawson, P. F. (1959). Individuals: An essay in descriptive metaphysics. London: Methuen.

Uller, C., Carey, S., Hauser, M., \& Xu, F. (1997). Is language needed for constructing sortal concepts? A study with non-human primates. In E. Hughes et al. (Eds.), Proceedings of the twenty-first Boston University conference on language development. Sommerville, MA: Cascadilla Press.

Van de Walle, G. A., Carey, S., \& Prevor, M. (2000). Bases for object individuation in infancy: Evidence from manual search. Journal of Cognition \& Development, 1(3), 249-280.

Wiggins, D. (1997). Sortal concepts: A reply to Xu. Mind and Language, 12(3-4), 413-421.

Wilcox, T., \& Baillargeon, R. (1998). Object individuation in infancy: The use of featural information in reasoning about occlusion events. Cognitive Psychology, 37(2), 97-155.

Wilcox, T., \& Chapa, C. (2002). Infants' reasoning about opaque and transparent occluders in an individuation task. Cognition, 85(1), B1-B10.

Wilcox, T., \& Schweinle, A. (2002). Object individuation and event mapping: Developmental changes in infants' use of featural information. Developmental Science, 5(1), 132-150.

Xu, F. (1997). Form Lot's wife to a pillar of salt: Evidence for physical object as a sortal concept. Mind and Language, 12, 365-392.

$\mathrm{Xu}, \mathrm{F}$. (2002). The role of language in acquiring object kind concepts in infancy. Cognition, 85(3), 223-250.

Xu, F., \& Baker, A. (2005). Object individuation in 10-month-old infants using a simplified manual search method. Journal of Cognition and Development, 6(3), 307-323.

Xu, F., \& Carey, S. (1996). Infants' metaphysics: The case of numerical identity. Cognitive Psychology, 30(2), 111-153.

$\mathrm{Xu}, \mathrm{F}$. , \& Carey, S. (2000). The emergence of kind concepts: A rejoinder to Needham and Baillargeon (2000). Cognition, 74(3), 285-301.

Xu, F., Carey, S., \& Quint, N. (2004). The emergence of kind-based object individuation in infancy. Cognitive Psychology, 49, 155-190.

Xu, F., Cote, M., \& Baker, A. (2005). Labeling guides object individuation in 12-month-old infants. Psychological Science, 16(5), 372-377. 